\section{FRI0145 BODY MASS DENSITY IN PATIENTS WITH SYSTEMIC LUPUS ERYTHEMATOSUS}

DM Obradovic, RM Stojanovic, G Susic. Clinical DEpartment VI, Institute of Rheumatology, Belgrade, Yugoslavia

10.1136/annrheumdis-2001.180

Background Physiological processes, as ageing and menopause, natural influence (diet, physical activity) and many other factors are important in development of osteopenia (OPE) or osteoporosis (OPO). The possible influence of some previous diseases and medications on bone density was investigated, too.

Objectives To estimate bone density in patient with Systemic Lupus Erythematosus (SLE) and to investigate the influence of BMI, age at onset of SLE, duration of disease, age in time of study, duration of CS treatment as a possible risk factors for OPE/OPO.

Methods In follow-up study we investigated 57 female patients with SLE, mean age $44.73 \pm 11.34$ years $(\min 22$-max68), mean age at the onset of SLE $35.83 \pm 11.18 \mathrm{yr}$.(15-64). The duration of SLE was $9.03 \pm 7.22$ yrs (1-32). Corticosteroide therapy was used for $8.40 \pm 6.85$ yr.(1-32). Body mass density was measured on L2-L4 segment of vertebral spine. DEXA LUNAR osteodensitometer was used. According to $\mathrm{T}$ score patients were classified as: normal ( $\mathrm{N}$ group) 21 patients $(\mathrm{T}>-1,0)$, osteopenic (OPE group) 23 pts $(-2.5<\mathrm{T}<-1.0)$, and ospeoporotic (OPO group) 13 pts $(\mathrm{T}<-2,5)$.

Results No difference were noted in: BMI and in duration of SLE in groups investigated. The borderline difference was existed between $\mathrm{N}$ and OPO group in duration of CS treatment $(p=0.049)$. The relation between particular data where statistical significance was obtained in groups investigated is presented in the Table 1.

\begin{tabular}{|c|c|c|c|}
\hline & $\mathrm{N}: \mathrm{OPE}$ & $\mathrm{N}: \mathrm{OPO}$ & OPE:OPO \\
\hline Age at onset of SLE & ns & $* * *$ & $* * *$ \\
\hline Age at the study & ns & $* * *$ & $* * *$ \\
\hline BMD & $* * *$ & $* * *$ & ns \\
\hline
\end{tabular}

Conclusion Low body mass density was found in elder group of patients with SLE, where the disease started later in perimenopause. Duration of disease, and duration of corticosteroid treatment did not influence BMD. Further studies will consider other factors possibly significant in development of osteoporosis in SLE (subsets of disease, immunological disturbances, cumulative dose of CS and known risk factors for osteoporosis.

\section{FRI0146 DEFORMING ARTHROPATHY OF THE HANDS (JACCOUD? S ARTHROPATHY) IN 20 PATIENTS WITH SYSTEMIC LUPUS ERYTHEMATOSUS (SLE)}

G Hayem, F Roux, SA Rouidi, E Palazzo, M Debandt, MF Kahn, 0 Meyer. Rheumatology Department, Bichat Hospital, Paris, France

\subsection{6/annrheumdis-2001.181}

Background Jaccoud?s arthropathy (JA) is a deforming but non erosive arthropathy that can affect patients with systemic lupus erythematosus (SLE).
Objectives To characterise SLE-associated JA (SLE-JA), and to compare the clinical and laboratory manifestations of SLE patients, with or without JA.

Methods From our cohort of 860 SLE patients, we selected all patients with clinical JA, as well as 86 patients without JA. Patients with erosive arthropathy were excluded. Clinical and laboratory manifestations were compared between the two groups.

Results We found 20 patients with SLE-JA (19 females, 1 male, all caucasians). Their mean age at SLE onset was $29.3 \pm 12.5$ yrs; mean duration of arthritis was $20.1 \pm 8.6$ yrs; mean number of 1982 ARA criteria for SLE was $5.9 \pm 1.5$ (range 4-9). Main deformations were ulnar drift (75\%), swan neck deformities (85\%), $\mathrm{Z}$ deformity of the thumbs (80\%) and boutonniere deformities (5\%). JA of the feet was present in 4 patients, with hallux valgus $(15 \%)$ or hammer toes $(5 \%)$ deformities. Major hyperlaxity of other joints was noted in 2 patients (10\%) and tendon rupture in 5 (25\%). Five patients underwent hand surgery for correction of deformities. Compared to SLE cases without JA, patients with SLE-JA had a higher frequency of Raynaud's phenomenon (50\% vs $15 \%$; p < 0.002) and IgM rheumatoid factor positivity ( $37 \%$ vs $15 \%$; p < 0.04), and a lower frequency of lymphopenia ( $45 \%$ vs $71 \%$; $<<0.03)$. No SLE-JA patient had antikeratin or antiperinuclear antibodies (ab). Anti-RA33 ab was absent in all 14 SLE-JA patients tested. The prevalence of other manifestations, including those related to antiphospholipid syndrome or SJÖGREN'S syndrome, was not different in the two groups.

Conclusion SLE-JA seems a rare condition. Rheumatoid factor inconstantly identifies this SLE subset, but the absence of other RA-associated $a b$, such as antifilaggrin $a b$, helps to differentiate SLE-JA from RA associated with SLE.

\section{FRI0147 BODY MASS DENSITY IN PATIENTS WITH SYSTEMIC LUPUS ERYTHEMATOSUS}

D Palic, R Stojanovic, G Susic. Clinical Department VI, Institute of Rheumatology, Belgrade, Yugoslavia

\subsection{6/annrheumdis-2001.182}

Background Physiological processes, as ageing and menopause, natural influence (diet, physical activity) and many other factors are important in development of osteopenia (OPE) or osteoporosis (OPO). The possible influence of some previous diseases and medications on bone density was investigated, too.

Objectives To estimate bone density in patient with Systemic Lupus Erythematosus (SLE) and to investigate the influence of BMI, age at onset of SLE, duration of disease, age in time of study, duration of CS treatment as a possible risk factors for OPE/OPO.

Methods In follow-up study we investigated 57 female patients with SLE, mean age 44,73 $\pm 11,34$ years $(\min 22$ max68), mean age at the onset of SLE $35.83 \pm 11.18$ yr.(15-64). The duration of SLE was $9.03 \pm 7.22$ yrs (1-32). Menarha were started in $13.73 \pm 1.47$ yr. (11-18). 27 pts were in menopause in 44.03 \pm 4.24 yr.(34-53). Corticosteroide therapy was used for $8.40 \pm$ 6.85 yr.(1-32). Body mass density was measured on L2-L4 segment of vertebral spine. DEXA LUNAR osteodensitometer was used. According to T score patients were classified as: normal (N group) 21 patients ( $\mathrm{T}>-1.0$ ), osteopenic (OPE group) 23 pts ($2,5<\mathrm{T}<-1.0$ ), and ospeoporotic (OPO group) 13 pts ( $\mathrm{T}<-$ $2.5)$. 